\title{
Acceptability of Contact Lenses Uses among Consumers in South Africa
}

\author{
Sandesh Srikissoon ${ }^{1}$ Prof Muhammad Hoque PhD $^{2}$
}

\begin{abstract}
${ }^{1}$ Post Graduate Student, Graduate School of Business and Leadership, University of KwaZulu-Natal, Durban, 4001, SOUTH AFRICA ${ }^{2}$ MANCOSA Graduate School of Business, 16 Samora Machel St, Durban Central, Durban, 4001, SOUTH AFRICA
\end{abstract}

\begin{abstract}
Advances in technology have resulted in contact lenses being more comfortable and able to correct myopia, hypermetropia and astigmatism. Also, contact lenses are useful for people with active lifestyles. Very little is known about the contact lens market in South Africa. Therefore, this study considered the profit margins in optometry and the viability of contact lenses as a means to increase revenue. The consumers were asked about their use of spectacles, lifestyles, awareness of contact lenses, and the benefits of using them. This was a cross-sectional study conducted among consumers. The findings revealed that many of the consumers found that spectacles were a hindrance in sporting activities and reported that given the opportunity, they would wear contact lenses. Half the respondents revealed that their optometrist discussed the contact lens option. Optometrists felt that actively prescribing contact lenses would be successful in increasing revenues. The study recommends that optometrists discuss the option of contact lenses with every patient, as this would ultimately result in increased profit margins and levels of patient satisfaction. Contact lens companies should also engage in marketing strategies to create awareness about the benefits of contact lenses for consumers.
\end{abstract}

Keywords: Consumer education; eye health; optometric industry.

\section{INTRODUCTION}

According to The Vision Impact Institute (2012), vision correction is required by 4.2 billion people at some stage of their lives, of which 1.7 billion receive spectacle or contact lenses. The balance of an estimated 2.5 billion people's vision remains uncorrected by these corrective lenses.

Contact lenses can correct short-sightedness (myopia), far-sightedness (hypermetropia) and astigmatism, the latter of which, according to the Mayo Foundation for Medical Education and Research (MFMER) (2020), is generally treatable and is when the curvature of the eye causes blurred and near vision. They advise that other conditions, such as headaches, difficulty with night vision, and eyestrain may also occur. When people over the age of forty require reading glasses they have a condition called presbyopia. Correction of the latter, according to the Mayo Foundation, may also occur with surgery. 


\section{LITERATURE REVIEW}

According to the FDA Food and Drug Administration USA (2018), there are two general categories of contact lenses-soft and rigid gas permeable (RGP). Contact lenses are currently more comfortable and able to be used for extended periods. They are available in a disposable form for yearly, monthly, bi-weekly and daily periods. The Mayo Foundation also advises that soft lenses can be worn continuously, even in sleep, for up to 30 days.

According to the British Contact Lens Association (2014), the following were identified as the main reasons to obtain contact lenses: Contact lenses offer natural all-round vision whilst enhancing your appearance. They are simple and convenient to use and expand lifestyle choices. Contact lenses have many advantages for sport and leisure activities. They can be worn every day, part-time or just for going out and special occasions. Vision with contact lenses can be as good as, or better than with spectacles. They offer a safe, effective, stable, and reversible alternative to refractive surgery. Contact lenses can correct almost all eyesight conditions and are suitable for people of all ages. Adaptation to contact lenses is rapid and trouble-free and the latest contact lenses and solutions provide excellent comfort and eye health. Some eye conditions can only be corrected with contact lenses, such as irregular astigmatism.

Atkins, Morgan and Morgan (2009) found in their experimental study that subjects appreciated the opportunity to try contact lenses before dispensing as one-third of the participants purchased contact lenses thereafter. These researchers recommended that practitioners consider offering this service to their clients and optimise the dispensing process.

Contact lenses have been proven to have various impacts at different stages of life. Pesudovs Garamendi and Elliot (2006) and Queirós, Villa-Collar, Gutiérrez, Jorge and González-Méijome (2012) found that participants' responses were highly favourable toward the lifestyle benefits of contact lenses. These included cosmetic benefits and contact lenses, which were found to be less restrictive during physically active tasks. In younger patients significantly greater lifestyle benefits have been noted as the self-perceptions of children improved by contact lens wear and fitting children with lenses does not require more time from the optometrist as compared to adults (Walline, Gaume, Jones, \& Rah, 2007; Rah, Walline, Jones-Jordan \& Sinnott, 2010; Anstice \& Phillips, 2011).

According to the Health Professions Council of South Africa (2014), there are 3527 registered optometrists in South Africa. Each optometry practice, specifically in the suburban areas, can only reach a threshold of a certain number of patients as market saturation and groups of practices increase the competition. From an internal perspective, rental (where applicable), staff salaries, and the cost of goods increase year on year. Ultimately, as optometrists graduate from universities and more optometry practices develop, more optometrists will compete for the same pool of customers. In many business environments where such is the case, net profits remain constant or shrink, while a select few can better compete and thereby grow profits.

The competition in the optometry industry is on the increase as with the advent of franchises, buyer power increases and many independent optometrists lose 'customers' to larger groups based on price and sometimes service. A segment of optometry that is increasing in developing countries is the contact lens segment. Some optometrists have devised a package which includes spectacles and contact lenses to make the concept more attractive to first-time wearers of contact lenses. The 
contact lens market appears to be a viable option to grow the optometric industry in South Africa. Therefore, the objective of this study was to investigate the acceptability of contact lenses uses among consumers in South Africa.

\section{RESEARCH METHODOLOGY}

A quantitative data was used in this study, which enabled a statistical analysis to allow for inferences to be drawn to a population. Minimum sample size for the study was calculated to be 385. A total of nine optometrists were selected randomly from the nine provinces to distribute the anonymous online questionnaire to their clients.

This questionnaire contained 17 questions. It was designed in the following manner:

- Three questions relating to demographic data

- Thirteen questions based on a scale

- One open-ended question

The three questions related to demographic data included the topics of gender, age group and the geographical location of the consumer.

The thirteen questions based on a scale pertained to the use of spectacles, the lifestyles of consumers, awareness of contact lenses, and the benefits of contact lenses. Those consumers who did not wear spectacles were asked to not proceed with the questionnaire as contact lenses would be of no benefit to them, while those who wore spectacles were asked to continue. Those consumers who wore spectacles and contact lenses were directed to questions related to the benefits of contact lenses such as, whether contact lenses have enhanced their lifestyles and if the associated benefits of contact lenses outweighed the cost. This was important as it indicated if consumers found benefits in using contact lenses and if they are willing to pay for that benefit. For those consumers who only wore spectacles, they were asked if given the option of contact lenses whether they would make a positive impact on their lifestyles and if they were aware of the benefits of wearing contact lenses and if they would wear contact lenses given the option. These questions were designed to determine whether those consumers are the potential target market for contact lens wear but have not been fully informed about contact lenses, or are not aware of the associated benefits of contact lenses.

The open-ended question was based on whether the consumer would wear contact lenses given the option. If the consumer answered no to this question, they were asked to provide a reason. This would provide insight into the reasons a spectacle wearer would not want to wear contact lenses.

\section{Data collection and analysis}

An electronic survey was created using Question Pro (an online survey tool). Questionnaires together with informed consent and covering letters were electronically mailed to the population of optometrists and population sample for the consumers. To ensure a better response rate, reminder emails were sent out to consumers in the third week following the initial email. .

Data from the survey questionnaires were coded and represented numerically by being captured on Microsoft Excel spreadsheets and processed in a graphical format. The descriptive and chisquare analyses were done. 


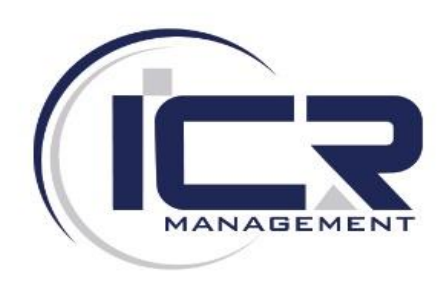

\section{RESULTS AND DISCUSSION}

A total of 481 people viewed the questionnaire, 283 people started the questionnaire, 245 people completed the questionnaire, and 38 people dropped out of the survey. Therefore, the completion rate of the survey was $51 \%$.

For the consumer population, the gender distribution was $52.24 \%$ females and $47.76 \%$ males, reflecting a relatively even spread for gender. Of the respondents, $86.16 \%$ were over the age of 25 -years-old, with $60.04 \%$ being over the age of 30-years-old. There were no respondents younger than the age of 15-years-old.

Respondents from all nine provinces completed the survey. The greatest number of respondents was recorded in KwaZulu-Natal (58.37\%) followed by Gauteng (21.63\%) and the Western Cape thereafter with $8.98 \%$. Of all the respondents, $67.76 \%$ experienced vision defects, while $32.24 \%$ responded as experiencing no visual defects.

Of the respondents, $53.06 \%$ responded as individuals who led an active lifestyle while $33.06 \%$ gave a neutral response to this question (Figure 1). Fewer than $15 \%$ of the respondents did not lead an active lifestyle. Of the respondents, $63.27 \%$ were currently spectacle wearers (Figure 2 ). The $36.73 \%$ of respondents who were not spectacle wearers were asked to stop after question six because if a person did not wear spectacles, they would not require corrective contact lenses.

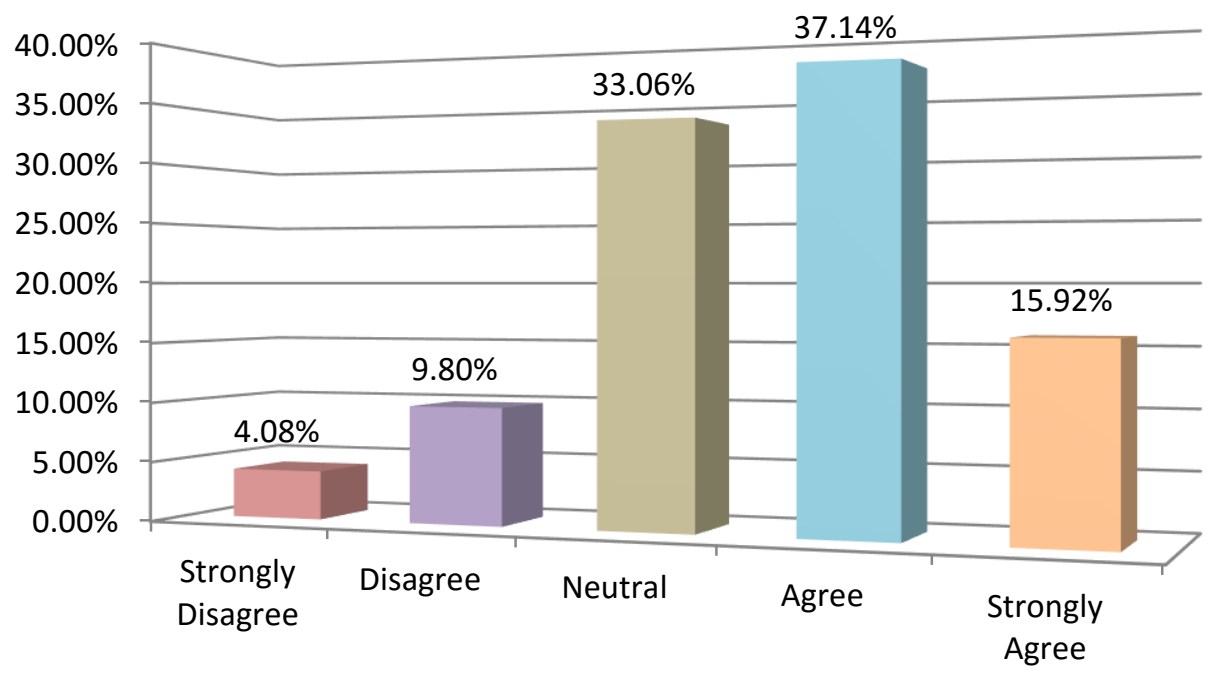

Figure 1: Active lifestyles 

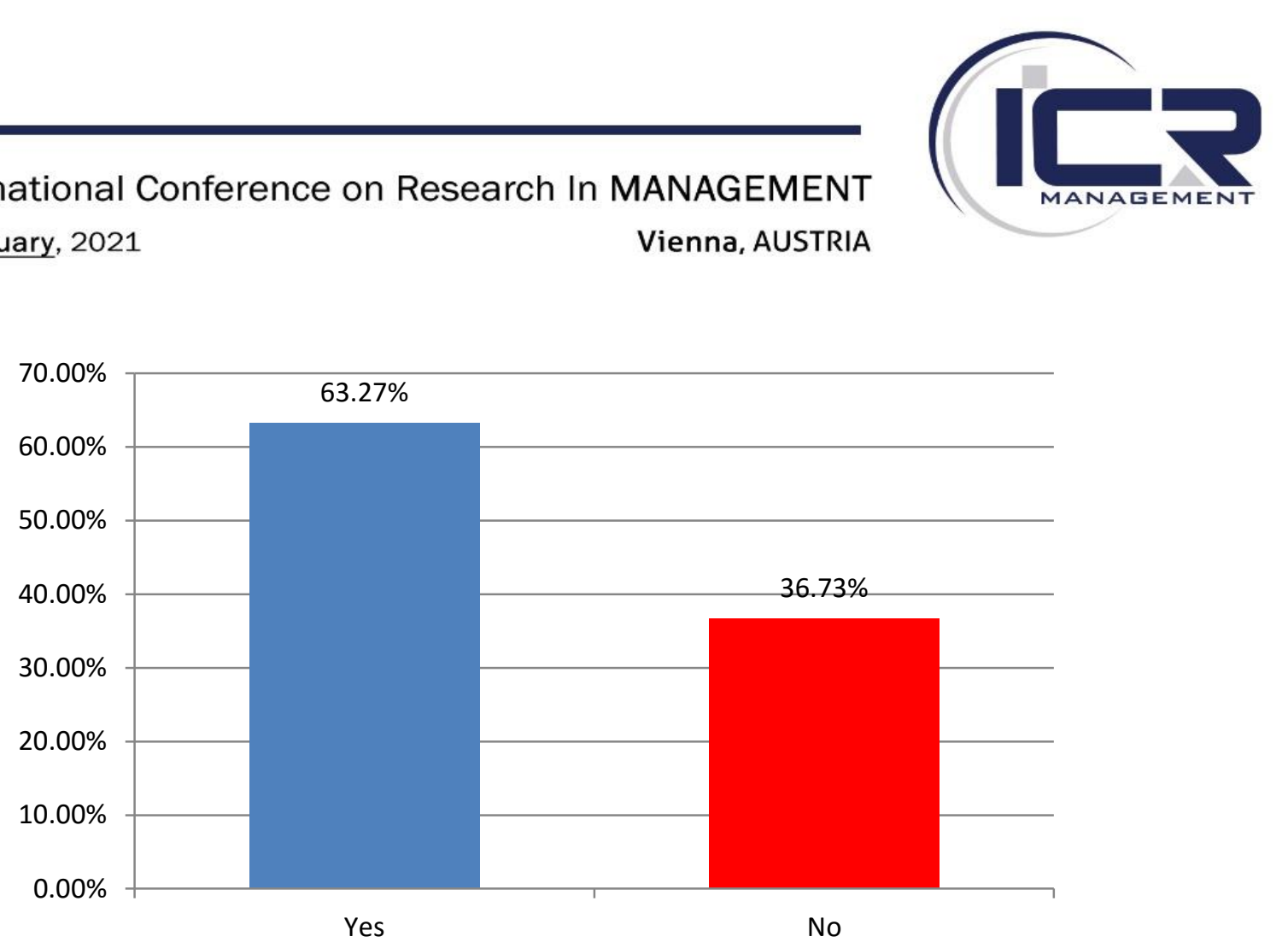

Figure 2: Percentage of consumers who wear spectacles

When asked if spectacles hinder their performance while playing sport or excelling at their hobbies, $66.45 \%$ of those respondents who wore spectacles responded by agreeing and strongly agreeing that spectacles hinder their performance while playing sport and excelling at their hobbies. $16.77 \%$ of the respondents gave a neutral response while $16.77 \%$ disagreed and strongly disagreed. According to the British Contact Lens Association (2014), one of the top ten reasons for wearing contact lenses is that it has numerous advantages for sport and leisure activities and can be used daily or part-time as required. $39.35 \%$ of the respondents who wore spectacles also wore contact lenses. When asked: Do you wear contact lenses? $60.65 \%$ of the respondents who wore spectacles did not wear contact lenses. Those respondents who wore contact lenses were asked to proceed to question 14 and answer the relevant questions, while those respondents who answered no were asked to continue till question 13.

Of the respondents, $54.26 \%$ who did not wear contact lenses but wore spectacle lenses reported that their optometrists had discussed the option of contact lenses with them. $45.74 \%$ of those respondents who wore spectacles and did not wear contact lenses reported that their optometrists did not discuss the option of contact lenses with them.

In response to the statement: Contact lenses could make a positive impact on your lifestyle, $46.80 \%$ of the respondents who wore spectacles and did not wear contact lenses responded by reporting that contact lenses could make a positive impact on their lifestyles. 34.04\% were neutral regarding this question while $15.96 \%$ disagreed and $3.19 \%$ strongly disagreed that contact lenses could make a positive impact on their lifestyles (Figure 3). According to the British Contact Lens Association (2014), contact lenses offer natural all-round vision while enhancing appearance. They are simple and convenient to use and contact lenses expand lifestyle choices. The British Contact Lens Association (2014), states that "Contact lenses can correct almost all eyesight conditions and are suitable for people of all ages". Thus, the possibility of contact lenses enhancing a person's lifestyle is indicated. Of the respondents who wore spectacles and did not wear contact lenses, 
$59.57 \%$ were aware of the benefits of wearing contact lenses, while $40.43 \%$ of respondents were not aware of the benefits of contact lenses.

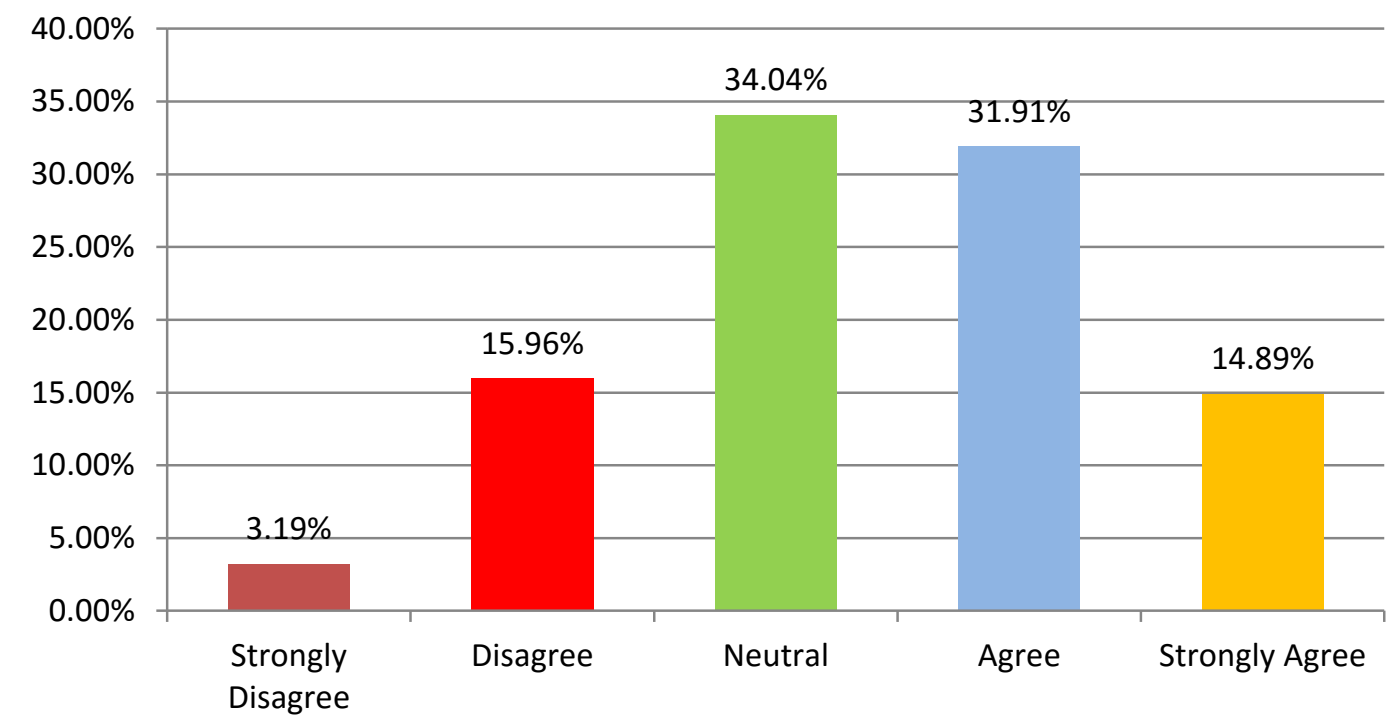

Figure 3: Positive impact of contact lenses on lifestyle

Of the respondents who wore spectacles but did not wear contact lenses, $56.38 \%$ responded by reporting that they would wear contact lenses if given the option. $43.62 \%$ responded by reporting that they would not wear contact lenses even if given the option

Those respondents who responded No to the question: Would you wear contact lenses if given the option, were asked to give reasons for stating so. The responses varied and were grouped as follows:

- Time-consuming to insert and remove contact lenses.

- Phobia of inserting contact lenses and having something on the eye.

- Phobia of infections because of contact lens usage.

- Sensitive eyes.

- People not being dependent on their spectacles all day, hence not finding the need for contact lenses.

These findings are in line with another study conducted by Wu, Carnt and Stapleton (2010) to investigate "Contact lens user profile, attitudes and level of compliance to lens care". The study concluded that poor hand hygiene, inadequate lens care, and not remembering when to return for aftercare are the common non-compliant behaviours in contact lens wearers.

Of the respondents who wore spectacles and contact lenses, 65.57\% responded by strongly agreeing and agreeing that contact lenses have enhanced their lifestyles (Figure 4). According to a study by Walline et al. (2007), contact lenses significantly improved the quality of life among children and teens as it improved their satisfaction with their vision correction and how they felt about their appearance and participated in activities. 
Of respondents who wore spectacles and contact lenses, $49.18 \%$ were of the view that contact lenses are expensive. $39.34 \%$ of respondents were neutral in this regard, while $11.48 \%$ of respondents disagreed and strongly disagreed that contact lenses were expensive.

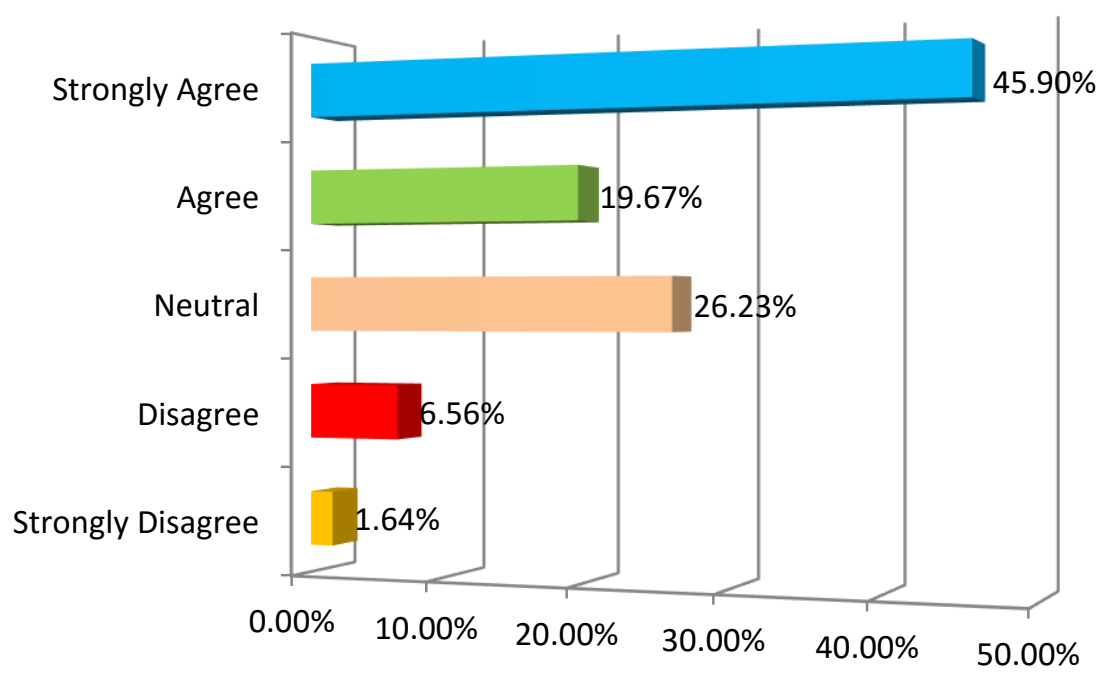

Figure 4: Contact lenses as a lifestyle enhancer

Of the respondents who wore spectacles and contact lenses, $47.55 \%$ agreed and strongly agreed that the benefits of contact lenses outweighed the associated cost of contact lenses. 39.34\% of the respondents gave a neutral response, while $13.12 \%$ of the respondents disagreed and strongly disagreed that the benefits of contact lenses outweigh the associated cost. Of the respondents who wore spectacles and contact lenses, 39.34\% disagreed and strongly disagreed that their spectacles are more comfortable than their contact lenses, while $36.07 \%$ of respondents agreed and strongly agreed that their spectacles are more comfortable than their contact lenses. According to the British Contact Lens Association (2014), vision with contact lenses can be as good, if not better than with spectacles as the contact lenses and contact lens solutions currently available provide excellent comfort and eye health thereby supporting the respondents who felt their contact lenses are more comfortable than their spectacles. 
2nd International Conference on Research In MANAGEMENT

19 _ 21 February, 2021

Vienna, AUSTRIA

Table 1: Cross-tabulation between would you wear contact lenses if given the option and age group

\begin{tabular}{|c|c|c|c|c|c|}
\hline \multicolumn{2}{|c|}{} & \multicolumn{2}{|c|}{$\begin{array}{c}\text { Would you wear contact lenses } \\
\text { if given the option }\end{array}$} & \multirow{2}{*}{ Chi-Square } & \multirow{2}{*}{ p-value } \\
\cline { 2 - 4 } & Yes & No & & \\
\hline & $15-20$ & $3(5.1 \%)$ & $2(4.3 \%)$ & & \multirow{2}{*}{7.831} \\
\cline { 2 - 4 } Age Group & $21-25$ & $8(13.6 \%)$ & $2(4.3 \%)$ & \multirow{2}{*}{0.05} \\
\cline { 2 - 4 } & $26-30$ & $12(20.3 \%)$ & $3(6.5 \%)$ & \\
\cline { 2 - 4 } & $>30$ & $36(61 \%)$ & $39(84.8 \%)$ & & \\
\hline
\end{tabular}

There was a significant association between would you wear contact lenses, if given the option, and the benefits of wearing contact lenses outweigh the associated cost $(\mathrm{p}=0.012)$. This meant that respondents who would wear contact lenses, if given the option, agree that the benefits of wearing contact lenses outweigh the associated cost (32.4\%), compared to those who would not wear contact lenses if given the option (8.3\%), (Table 2) to establish if consumers will use and purchase contact lenses if given the option.

Table 2: Cross-tabulation between would you wear contact lenses if given the option and the benefits of contact lenses outweigh the associated cost

\begin{tabular}{|c|c|c|c|c|c|}
\hline & & \multicolumn{2}{|c|}{$\begin{array}{c}\text { Would you wear contact lenses if } \\
\text { given the option }\end{array}$} & \multirow{2}{*}{ Chi-Square } & \multirow{2}{*}{ p-value } \\
\hline & & Yes & No & & \\
\hline \multirow{3}{*}{$\begin{array}{l}\text { The benefits of } \\
\text { contact lenses } \\
\text { outweigh the } \\
\text { associated cost }\end{array}$} & Disagree & $4(11.8 \%)$ & $10(41.7 \%)$ & \multirow{4}{*}{8.924} & \multirow{4}{*}{0.012} \\
\hline & Neutral & $19(55.9 \%)$ & $12(50.0 \%)$ & & \\
\hline & Agree & $11(32.4 \%)$ & $2(8.3 \%)$ & & \\
\hline \multicolumn{2}{|c|}{ Total } & $34(100.0 \%)$ & $24(100.0 \%)$ & & \\
\hline
\end{tabular}

\section{CONCLUSION AND RECOMMENDATIONS}

The majority of those respondents who wore spectacles and did not wear contact lenses reported that their optometrists did not discuss the option of contact lenses with them. This is significant as the majority of spectacle lens prescriptions are available for contact lens prescriptions and thus highlights the potential market that is not utilized by optometrists. Of the respondents who wore 
spectacles and did not wear contact lenses, the majority responded by reporting that contact lenses could make a positive impact on their lifestyles. This is also a significant number, as it indicates consumers' willingness towards contact lenses as an aid to vision. Almost three-fifth (59.57\%) of non-contact lenses wearers were aware of the benefits of contact lenses, thus showing that by consumers being aware of the benefit of contact lenses, they are more likely to wear contact lenses if given the option. Several respondents who only wore spectacles responded that they would wear contact lenses if given the option. From these responses, it can be deduced that more consumers are willing to try contact lenses if given the option.

Of the respondents who wore spectacles and contact lenses, $65.57 \%$ responded by strongly agreeing and agreeing that contact lenses have enhanced their lifestyles. It can be concluded that even though respondents viewed contact lenses as expensive, respondents are still willing to purchase contact lenses as more respondents believed that the associated benefits of contact lenses outweigh the cost. The responses to comfort levels of contact lenses and spectacles highlight that consumers who wear both spectacles and contact lenses have similar levels of comfort with each vision aid.

There was a statistically significant association to the responses from: Would you wear contact lenses if given the option and the age group of the respondents. When comparing the responses to: Would you wear contact lenses if given the option to the responses to: The benefits of wearing contact lenses outweigh the associated cost, a statistically significant relationship existed. The majority of respondents who would wear contact lenses if given the option were under the age of 30 -years-old and the majority of the respondents who would wear contact lenses if given the option believed that the benefits of wearing contact lenses outweigh the associated cost.

From the discussion above, it can be concluded that more consumers are willing to use and purchase contact lenses if given the option.

\section{Recommendations and future research}

The study revealed that optometrists are of the view that profit margins are likely to decrease over time. It was also found that more optometrists did not actively prescribe contact lenses as a means to increase revenues, while optometrists feel that actively prescribing contact lenses would be successful. The third research objective of the study showed that more consumers are willing to use and purchase contact lenses if given the option, thus from the research, it is recommended that optometrists discuss the option of contact lenses with every patient as this would ultimately result in increased profit margins and increased levels of patient satisfaction, which would lead to greater repeat business from satisfied consumers.

Another recommendation would be that contact lens companies engage in marketing strategies that create awareness about the benefits of contact lenses for consumers. This could be done through television, radio and suitable print media. From the results of the consumer survey, many consumers are of the view that contact lenses could be harmful to their eye health. Therefore, it rests with manufacturers and optometrists to better educate consumers on the benefits of contact lenses that are currently available as technological advancements in these devices have made it possible to eliminate many of the complications associated with older contact lenses. 
Minimal research has been conducted in the contact lens market in South Africa, and it is, therefore, recommended that further studies in South Africa address this shortfall. The total volume of spectacle lenses sold to consumers should be compared to the total volume of contact lenses sold related to the volume of consumers who wear spectacle lenses and those who wear contact lenses thereby identifying the gap that exists between spectacle wearers and contact lens wearers. This would show the potential market space available for contact lenses as a vehicle to grow the optometric industry in South Africa.

\section{Conclusion}

The results of the surveys provided an interpretation of the data using descriptive statistics and statistical analysis which showed associations where possible. Descriptive statistics allowed for the graphical representation of the responses to the questionnaires, while chi-squared analysis was used to show associations of the responses. It provided analysis and discussion of the results of the surveys using literature where available to explain possible reasons for the responses received.

While a limitation in terms of responses from certain provinces existed, those respondents who responded provided a wealth of information and insight into the perception of contact lenses as a business driver in the optometric industry of South Africa. Some optometrists who participated in the study requested that a summary of findings be sent to them on completion of the study. This study concluded that contact lenses have the potential to grow the optometric industry in South Africa.

\section{REFERENCES}

Anstice, N.S. \& Phillips, J.R. Effect of dual-focus soft contact lens wear on axial myopia progression in children. Ophthalmology. 2011;118:152-161. https://doi.org/10.1016/j.ophtha.2010.10.035.

Atkins, N., Morgan, S.L. \& Morgan, P.B. Enhancing the approach to selecting eyewear (EASE): a multi-centre, practice-based study into the effect of applying contact lenses prior to spectacle dispensing. Contact Lens \& Anterior Eye, Vol. 32. 2009:103-107. DOI: $10.1016 /$ j.clae.2009.02.004.

British Contact Lens Association (2014). Available at: https://www.bcla.org.uk/

FDA U.S. Food and Drug Administration. Types of contact lenses. 2018. [Online] Available: https://www.fda.gov/medical-devices/contact-lenses/types-contact-lenses; [Accessed: 10 July 2019].

Mayo Foundation for Medical Education and Research (MFMER) Presbyopia. 2020. [Online] Available: https://www.mayoclinic.org/diseases-conditions/presbyopia/symptoms-causes/syc20363328; [Accessed: 20 March 2019].

Health Professions Council of South Africa (2014). Available at: https://www.hpcsa.co.za/?contentId=462\&menuSubId=0\&actionName=Home

Pesudovs, K., Garamendi, E. \& Elliot, D.B. A quality of life comparison of people wearing spectacles or contact lenses or having undergone refractive surgery. Journal of Refractive Surgery, Vol. 22;2006:19-27. PMID: 16447932. 
Queiros, A., Villa-Collar C, Gutiérrez AR, Jorge J, González-Méijome JM. Quality of life of myopic subjects with different methods of visual correction using the NEI RQL-42 questionnaire. Eye Contact Lens. 2012 Mar,38;2012:116-121.

Rah, M.J., Walline, J.J., Jones-Jordan, L.A., Sinnott, L.T., et al. Vision specific quality of life of pediatric contact lens wearers. Optometry and Vision Science, Vol. 87;2010:560-566. doi: 10.1097/OPX.0b013e3181e6a1c8.

The Vision Impact Institute (2012). Available at: https://visionimpactinstitute.org/vision-research/

Walline, J.J., Gaume, A.O.D., Jones, L.A. \& Rah M. Et al. Benefits of contact lens wear for children and teens. Eye Contact Lens, Vol. 33;2007:317-321. doi: 10.1097/ICL.0b013e31804f80fb.

Wu, Y., Carnt, N. \& Stapleton, F. Contact lens user profile, attitudes and level of compliance to lens care. Contact Lens \& Anterior Eye, Vol. 33;2010:183-188. https://doi.org/10.1016/j.clae.2010.02.002. 